\title{
Comparison of the Effectiveness and Safety of Vildagliptin Add-On to Metformin Versus Other Oral Dual Antidiabetes Agents in Patients with Type 2 Diabetes: The China Prospective Diabetes Study
}

\author{
Li Zang • Yin Han · Lixian Chen · Daqing Hu • Hui Jin • \\ Nailong Yang · Xiaoyun Shi · Linlang Liang · Mingming Liu • \\ Hong Fan · Quanmin Li · Yiming Mu
}

Received: May 9, 2019 / Published online: June 20, 2019

(C) The Author(s) 2019

\section{ABSTRACT}

Introduction: The efficacy and safety of vildagliptin alone or with metformin is well established by randomized trials, but it is unknown whether it can be extrapolated to the real-world setting in Chinese patients with type 2 diabetes mellitus (T2DM). This study aimed to assess the

Enhanced Digital Features To view enhanced digital features for this article go to https://doi.org/10.6084/ m9.figshare.8210072.

Electronic supplementary material The online version of this article (https://doi.org/10.1007/s13300$019-0645-\mathrm{z}$ ) contains supplementary material, which is available to authorized users.

L. Zang · Y. Mu ( $₫)$

Department of Endocrinology, Chinese PLA General Hospital, Beijing, China

e-mail: muyiming@301hospital.com.cn

Y. Han

Department of Endocrinology, Tianjin Haibin

People's Hospital, Tianjin, China

L. Chen

Department of Endocrinology, Tianjin Hexi Ruijing

Metabolic Disease Hospital, Tianjin, China

D. $\mathrm{Hu}$

Department of Endocrinology, Wuhan Asia Heart

Hospital, Wuhan, China

H. Jin

Department of Endocrinology, Zhongda Hospital

Southeast University, Nanjing, China effectiveness and safety of vildagliptin add-on to metformin versus dual oral antidiabetes drug (OAD), non-vildagliptin combination therapies in real-world Chinese patients with T2DM.

Methods: The China Prospective Diabetes Study was a post-marketing, prospective, multicenter, observational, real-world study conducted in 52 centers. Patients inadequately controlled with OAD monotherapy and who initiated vildagliptin add-on to metformin (VM cohort) or two OADs other than vildagliptin (comparator cohort) were included for the present analysis. The composite primary endpoint was glycated hemoglobin (HbA1c) $<7 \%$ and without tolerability events (hypoglycemia,

\section{N. Yang}

Department of Endocrinology, The Affiliated

Hospital of Qingdao University, Qingdao, China

\section{Shi}

Department of Endocrinology, The Third Medical

Center of Chinese PLA General Hospital, Beijing, China

\section{Liang}

Department of Endocrinology, General Hospital of Northern Theater Command of Chinese PLA, Shenyang, China

\section{Liu - H. Fan}

Novartis Pharma AG, Beijing, China

Q. Li ( $\square)$

Department of Endocrinology, Rocket Army

Medical Center of Chinese PLA General Hospital,

Beijing, China

e-mail: liqm0806@163.com 
weight gain $\geq 3 \%$, or discontinuation due to gastrointestinal events) at 12 months. Secondary endpoints included change in $\mathrm{HbA} 1 \mathrm{c}$ from baseline, subgroup analysis, and tolerability. Propensity score matching analysis was performed to adjust for baseline covariates imbalance (body mass index (BMI) and HbA1c).

Results: A total of 604 patients received VM and 670 received comparator therapy. Patients who received VM were younger, more obese, and had a higher baseline HbA1c and a shorter duration of T2DM. After propensity score matching, there were 530 patients per cohort. After 12-month treatment, the success rates of the composite primary endpoint were $50.9 \%$ and $33.0 \%$ in the $\mathrm{VM}$ and comparator cohorts, respectively $(P<0.001$; odds ratio $=2.10,95 \%$ confidence interval (CI) 1.64-2.70). Furthermore, the success rates of the composite endpoint were higher with VM across geographic area, BMI, and baseline HbA1c subgroups. Fewer tolerability events occurred in the VM cohort versus the comparator cohort $(8.3 \%$ vs. $16.2 \%, P<0.001$; relative risk $=0.51,95 \%$ CI $0.36-0.72$ ).

Conclusion: Compared with dual OAD nonvildagliptin combination therapies, vildagliptin add-on to metformin is effective and safe to achieve glycemic control in Chinese patients with T2DM.

Funding: Novartis.

Keywords: Add-on therapy; Dual oral antidiabetes drugs; Effectiveness; Real world; Safety; Vildagliptin

\section{INTRODUCTION}

China has the world's largest type 2 diabetes mellitus (T2DM) population (114.4 million), with an estimated overall prevalence of $10.9 \%$ reported in Chinese adults in 2017 [1]. Despite the availability of several antidiabetes drugs, only $47.7 \%$ of patients with T2DM achieve the target glycated hemoglobin (HbA1c) levels $(<7 \%)$ in China [2]. The China DiaSTAGE study suggested that poor target achievement in Chinese patients with T2DM $>65$ years of age could be due, at least in part, to age, duration of T2DM, body mass index (BMI), HbA1c control, and hypoglycemia events; meanwhile, inadequate glycemic control in these patients might also be due to the use of insulin secretagogues [3].

At present, lifestyle modifications and metformin are the first-line treatment strategies for T2DM [4]. Nevertheless, studies have shown that there is a progressive loss of glycemic control with prolonged use of metformin, leading to unsatisfactory long-term outcomes $[5,6]$. The 2017 Chinese Guidelines for the Prevention and Treatment of T2DM emphasized the importance of using two oral antidiabetes drugs (OADs) [7]. The 2019 American Diabetes Association Guideline also suggested that two OADs should be initially administered in patients with newly diagnosed T2DM and HbA1c levels > 9\% [4]. In addition, the 2019 American Association of Clinical Endocrinologists (AACE)/American College of Endocrinology (ACE) T2DM management guidelines suggested that combination therapy of metformin with other OADs such as glucagon-like peptide 1 (GLP-1) agonists, sodium-dependent glucose transporter 2 (SGLT2) inhibitors, dipeptidyl peptidase-4 (DPP-4) inhibitors, thiazolidinediones (TZD), insulin, alpha-glycosidase inhibitors, sulfonylureas, or glinides should be considered in selected patients on an individual basis [8]. Hence, it is important to consider the addition of a second OAD for the optimization of glycemic control, but the risk of adverse events (such as hypoglycemia and weight gain) has to be carefully weighed against the benefits.

Vildagliptin is a potent and selective DPP-4 inhibitor that increases $\alpha$ - and $\beta$-cell responsiveness to glucose, without weight gain or increased risk of hypoglycemia $[9,10]$. The efficacy and safety of vildagliptin alone or with metformin have been well established in randomized controlled trials (RCTs) in predominately Caucasians $[11,12]$, but it is still unknown whether these findings can be extrapolated to the real-world setting in Chinese patients with T2DM.

The China Prospective Diabetes Study (China PDS) is a post-marketing, prospective, multicenter, observational, real-world study designed to evaluate the effectiveness and safety of vildagliptin add-on to metformin for the treatment of T2DM patients in China. 


\section{METHODS}

\section{Study Design and Subjects}

This study was conducted between June 2013 and April 2017 in 52 centers of China. The study was approved by the ethics committees of all participating institutions (Supplementary Table 1). Chinese PLA General Hospital piloted the ethical review of this study. Informed consent for the study was obtained from all participants. The study was conducted in compliance with the Helsinki Declaration of 1964 and its later amendments. The eligibility criteria were (1) age $\geq 18$ years; (2) diagnosed with T2DM; 3 ) recent ( $<3$ months) HbA1c measurement between $7 \%$ and $11 \%$; (4) received oral monotherapy with sulfonylurea, metformin, TZD, or alpha-glycosidase inhibitors; and (5) plan to initiate a second OAD on the basis of the local physician's decision. Patients were excluded from the study if they (1) had participated in any RCT; (2) had been treated with insulin and/ or GLP-1 analogue or agonist, and/or OAD single-pill combination (SPC), or planned to initiate any of the aforementioned drugs after enrollment; (3) were diagnosed with type 1 diabetes mellitus; (4) were pregnant or lactating; or (5) were unable to undergo regular follow-up on the basis of the investigator's judgment, including non-resident and other factors.

The local physician chose the OADs at their discretion. To ensure the non-interventional status of the study, patient enrollment was carried out after the treatment decision had been made. Among all patients enrolled in the China PDS, patients initiated treatment with vildagliptin add-on to metformin (VM cohort) or any two OADs other than vildagliptin (comparator cohort) were included in the present analysis.

\section{Data Collection}

Data were recorded using an electronic case report form (eCRF) and collected by using an electronic data capture (EDC) system. Medications were recorded in the database by using the World Health Organization (WHO) drug reference directory. Adverse events (AEs) were recorded by using Medical Dictionary for Regulatory Activities (MedDRA) version 15.1.

The patient demographic characteristics included age, gender, BMI, ethnicity, baseline HbA1c, duration of T2DM, complications, smoking history, and T2DM family history.

\section{Follow-up and Endpoints}

The composite primary endpoint was defined as good glucose control (HbA1c $<7 \%$ ) without any tolerability event after 12 months of treatment (i.e., treatment success). The tolerability events included hypoglycemia (presence of symptoms suggestive of hypoglycemia, confirmed by self-monitored glucose $<3.1 \mathrm{mmol} / \mathrm{L}$, and symptom relief after consuming carbohydrates [13]), clinically significant weight gain (weight gain $\geq 3 \%$ based on National Institutes of Health (NIH) guidelines [14, 15]), and discontinuation due to gastrointestinal (GI) events (such as decreased or increased appetite, abdominal pain, diarrhea, nausea, vomiting, dry mouth, dyspepsia, and weight loss [16]).

The secondary endpoints were (1) the mean changes in $\mathrm{HbA1c}$ from baseline to 3,6 , and 12 months; (2) glucose control (HbA1c $<7 \%)$ at 3,6 , and 12 months; (3) safety and tolerability; (4) the success rate of the composite endpoint according to the geographic region (eastern China, northern China, and southern China), BMI subgroups (BMI $<24,24-28$, and $>28 \mathrm{~kg} /$ $\mathrm{m}^{2}$ ), baseline HbA1c subgroups (HbA1c $<8 \%$, $8-9 \%$, and $>9 \%)$, the non-prespecified subgroups (including age, gender, duration of T2DM, T2DM complication, smoking history, and T2DM family history); (5) the success rate of the composite endpoint and the proportion of patients achieving HbA1c $<7 \%$ after 3, 6, and 12 months in the VM cohort versus insulinsecreting agent add-on to metformin (IM) subgroup of the comparator cohort; and (6) the success rate of the composite endpoint and the proportion of patients achieving HbA1c $<7 \%$ after 3,6 , and 12 months in the VM cohort versus $\alpha$-glucosidase inhibitor add-on to metformin (AM) subgroup of the comparator cohort. 
The safety outcomes, as evaluated by vital sign, physical and lab examinations, included all AEs recorded during OAD treatment in the VM and comparator cohorts.

\section{Statistical Analysis}

Sample size calculation was based on the success rates of the composite primary endpoint in the VM cohort (34.0\%) and comparator cohort (21.3\%) (estimation based on the EDGE study [17]). The two-sided $Z$ test was used, with $\alpha=0.05$ and $\beta=90 \%$. At a dropout rate of $20 \%$, 321 subjects were required for the VM cohort and 217 subjects were required for the comparator cohort.

The full analysis set (FAS) included all patients in the VM or comparator cohorts with at least one effectiveness evaluation after baseline and before therapy change. The safety analysis set (SAS) included all patients who participated in this study and had at least one safety evaluation after baseline.

Propensity score matching (PSM) was carried out to adjust for baseline covariates between the $\mathrm{VM}$ and comparator cohorts on the basis of BMI and HbA1c.

Continuous variables are presented as means and standard deviations (SD) and were analyzed using the Mann-Whitney $U$ test. Categorical variables are presented as frequencies and were analyzed using the chi-square test. $P$ values $<0.05$ were considered statistically significant on the basis of two-tailed tests. Odds ratio (OR) was used to compare treatment success and relative risk (RR) was used to compare treatment safety between the two cohorts; ORs and RRs are presented with the corresponding 95\% confidence intervals (CIs). All statistical analyses were performed using SAS 9.2 (SAS Institute, Cary, NY, USA).

\section{RESULTS}

\section{Baseline Characteristics}

From June 2013 to April 2017, 1657 patients were enrolled. After exclusion of patients with eligibility violation $(n=2)$ and no follow-up data $(n=160)$, a total of 1495 patients were included for analysis. The SAS included 724 patients in the VM cohort and 771 in the comparator cohort. The FAS included 604 patients in the VM cohort and 670 in the comparator cohort (Fig. 1). After PSM, there were $530(87.7 \%)$ patients in the VM cohort and $530(79.1 \%)$ in the comparator cohort. The mean follow-up period was $12 \pm 2$ months. The detailed OAD treatment regimens prescribed in the real-life setting are shown in Supplementary Table 2. The most common vildagliptin dual therapies were vildagliptin plus metformin $(64.7 \%)$, followed by vildagliptin plus sulfonylurea (SUs 14.7\%) and vildagliptin plus $\alpha$-glucosidase inhibitor (13.0\%). The most common dual therapies excluding vildagliptin were metformin plus SUs (37.8\%) and metformin plus $\alpha$-glucosidase inhibitor (23.7\%).

The demographic characteristics of the patients before and after PSM are shown in Table 1. Before PSM, age was $51.8 \pm 11.0$ years for the VM cohort and $57.7 \pm 12.5$ years for the comparator cohort $(P<0.001)$. The proportion of male patients (61.9\% vs. 56.3\%, $P<0.05$ ), baseline HbA1c levels $(8.32 \pm 1.05 \%$ versus $8.06 \pm 0.92 \%, P<0.001)$, and BMI $\left(26.4 \pm 3.9 \mathrm{~kg} / \mathrm{m}^{2}\right.$ vs. $25.3 \pm 3.4 \mathrm{~kg} / \mathrm{m}^{2}$, $P<0.001)$ were higher in the VM cohort. The duration of T2DM in the VM cohort was shorter than that in comparator cohort $(50.6 \pm 55.1$ months vs. $72.8 \pm 66.3$ months, $P<0.001)$. After PSM, there were no significant differences in the baseline $\mathrm{HbA1c}(8.17 \pm 0.96 \%$ vs. $8.20 \pm 0.95 \%)$ and BMI $\left(25.9 \pm 3.1 \mathrm{~kg} / \mathrm{m}^{2}\right.$ vs. $\left.25.8 \pm 3.1 \mathrm{~kg} / \mathrm{m}^{2}\right)$ between the VM and comparator cohorts $(P>0.05)$.

\section{Composite Endpoint}

Results of the composite primary endpoint and individual components of the composite endpoint after PSM are shown in Fig. 2. After 12 months of treatment, the success rate of the composite primary endpoint in the VM cohort was higher than that in the comparator cohort (50.9\% vs. $33.0 \%, P<0.001$; OR $2.10,95 \% \mathrm{CI}$ 1.64-2.70) (Fig. 2a). At 3 months, there was no statistical difference in the proportion of 


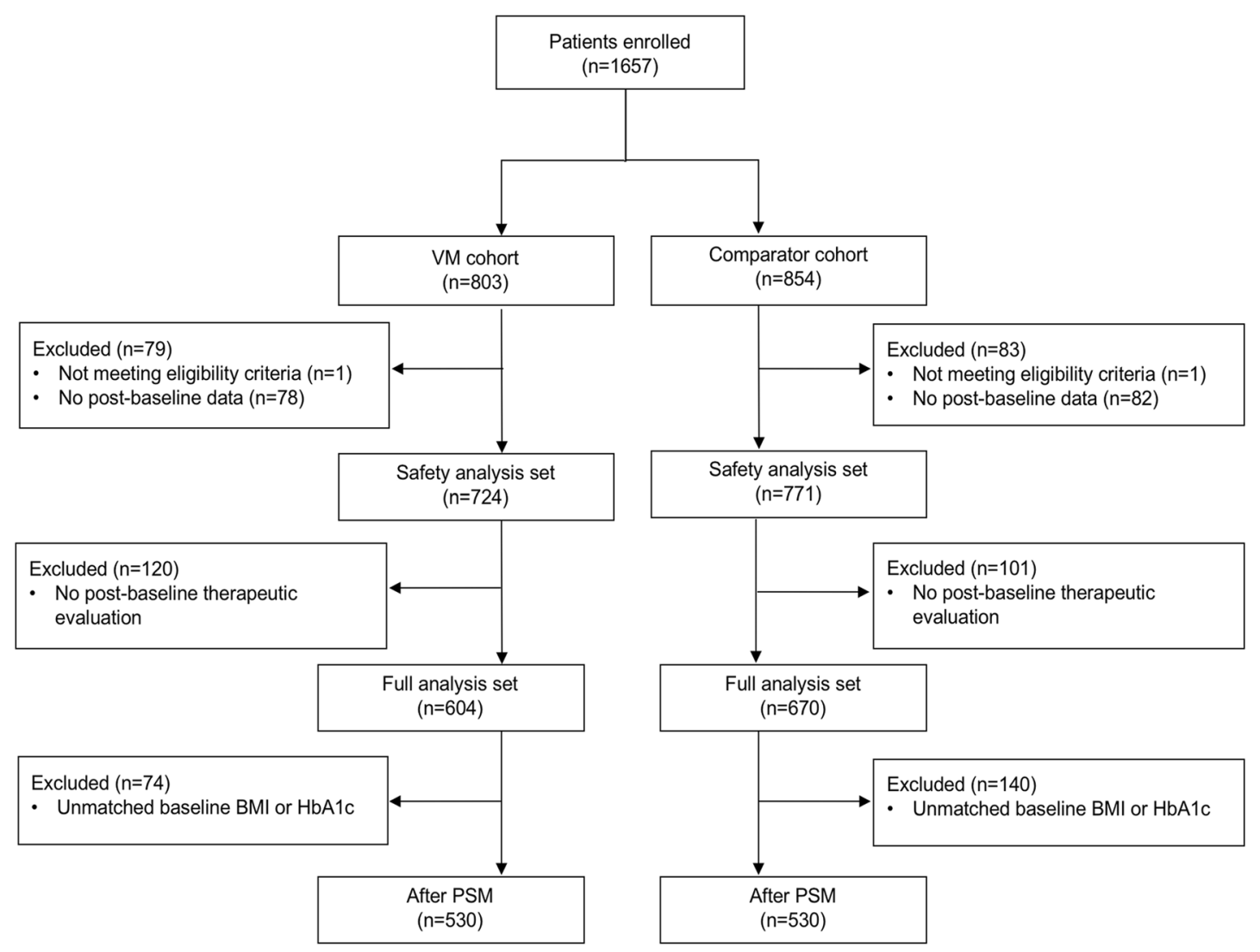

Fig. 1 Flowchart

patients with $\mathrm{HbA} 1 \mathrm{c}<7 \%$ between the two cohorts. This proportion showed no significant changes in the comparator cohort, but steadily increased thereafter in the VM cohort. At 6 months, the proportion of patients reaching HbA1c $<7 \%$ was higher than that in comparator cohort $(51.5 \%$ vs. $43.1 \%, P<0.01)$. At 12 months, the proportion of patients with $\mathrm{HbA} 1 \mathrm{c}<7 \%$ in the VM cohort $(54.3 \%)$ was significantly higher than that in the comparator cohort $(41.7 \%)(P<0.001)$ (Fig. $2 b)$.

The mean changes in HbA1c in the VM and comparator cohorts at 3, 6, and 12 months from baseline after PSM are shown in Supplementary Fig. 1. At 6 and 12 months, the decrease in HbA1c from baseline in the VM cohort was more pronounced than that in the comparator cohort ( 3 months: $-1.12 \pm 1.18 \%$ vs. $-0.95 \pm$ $1.24 \%, P<0.01 ; 6$ months: $-1.14 \pm 1.29 \%$ vs. $-0.89 \pm 1.31 \%, P<0.001)$.
The tolerability profile of the VM and comparator cohorts after PSM are shown in Fig. 2c. The occurrence rate of total tolerability events in the VM cohort $(8.3 \%, 44 / 530)$ was lower than in the comparator cohort $(16.2 \%, 86 / 530)$ after 12 months of treatment (RR $0.51,95 \% \mathrm{CI}$ $0.36-0.72)$. The rate of weight gain $\geq 3 \%$ in the VM cohort $(7.9 \%, 42 / 530)$ was also lower than in the comparator cohort $(14.2 \%, 75 / 530)$ (RR $0.56,95 \%$ CI $0.39-0.80)$. The occurrence rate of hypoglycemic events in the VM cohort $(0.4 \%$, $2 / 530$ ) was lower than in the comparator cohort $(2.3 \%, 12 / 530)$ (RR 0.17, 95\% CI 0.04-0.74).

\section{Subgroup Analyses}

Results of the composite endpoint from the prespecified subgroups analysis before PSM are shown in Fig. 3. Among the three geographic region subgroups, the success rates of the composite endpoint with VM in eastern China (OR 
Table 1 Demographic characteristics of patients before and after PSM (full analysis set)

\begin{tabular}{|c|c|c|c|c|c|c|}
\hline \multirow[t]{2}{*}{ Parameter } & \multicolumn{3}{|c|}{ Matched cohort } & \multicolumn{3}{|l|}{ Full cohort } \\
\hline & $\begin{array}{l}\text { VM } \\
(n=530)\end{array}$ & $\begin{array}{l}\text { Comparator } \\
(n=530)\end{array}$ & $P$ & $\begin{array}{l}\mathrm{VM} \\
(n=604)\end{array}$ & $\begin{array}{l}\text { Comparator } \\
(n=670)\end{array}$ & $P$ \\
\hline Age, years & $52.0 \pm 10.8$ & $57.1 \pm 12.2$ & $<0.001$ & $51.8 \pm 11.0$ & $57.7 \pm 12.5$ & $<0.001$ \\
\hline Gender (male), $n \%$ & $323(60.9)$ & $306(57.7)$ & 0.288 & $374(61.9)$ & $377(56.3)$ & 0.041 \\
\hline BMI, $\mathrm{kg} / \mathrm{m}^{2}$ & $25.9 \pm 3.1$ & $25.8 \pm 3.1$ & 0.647 & $26.4 \pm 3.9$ & $25.3 \pm 3.4$ & $<0.001$ \\
\hline Ethnicity (Han), $n \%$ & $517(97.5)$ & $515(97.2)$ & 0.120 & $589(97.5)$ & $653(97.5)$ & 0.466 \\
\hline Baseline HbA1c, \% & $8.17 \pm 0.96$ & $8.20 \pm 0.95$ & 0.521 & $8.32 \pm 1.05$ & $8.06 \pm 0.92$ & $<0.001$ \\
\hline $\begin{array}{l}\text { Duration of T2DM, } \\
\text { months }\end{array}$ & $52.7 \pm 55.9$ & $69.7 \pm 63.2$ & $<0.001$ & $50.6 \pm 55.1$ & $72.8 \pm 66.3$ & $<0.001$ \\
\hline Complications, $n \%$ & $251(47.4)$ & $237(44.7)$ & 0.388 & $322(53.3)$ & $353(52.7)$ & 0.823 \\
\hline Smoking history, $n \%$ & $141(26.6)$ & $134(25.3)$ & 0.668 & $172(28.5)$ & $168(25.1)$ & 0.254 \\
\hline $\begin{array}{l}\text { T2DM family history, } \\
n \%\end{array}$ & $216(40.8)$ & $216(40.8)$ & 0.393 & $243(40.2)$ & $270(40.3)$ & 0.606 \\
\hline
\end{tabular}

Data are $n(\%)$ or mean \pm SD

$V M$ vildagliptin add-on to metformin, $B M I$ body mass index, $H b A 1 c$ glycated hemoglobin, $T 2 D M$ type 2 diabetes mellitus

2.47, 95\% CI 1.57-3.89), northern China (OR $1.69,95 \%$ CI 1.18-2.42), and southern China (OR 3.43, 95\% CI 1.22-9.67) were higher than with comparator.

Among the three BMI subgroups, the success rates of the composite endpoint with $\mathrm{VM}$ in the $\mathrm{BMI}<24 \mathrm{~kg} / \mathrm{m}^{2}$ (OR 1.65, 95\% CI 1.08-2.50), $24-28 \mathrm{~kg} / \mathrm{m}^{2}$ (OR 2.22, 95\% CI $1.61-3.07$ ), and $>28 \mathrm{~kg} / \mathrm{m}^{2}$ (OR 2.02, 95\% CI 1.22-3.37) subgroups were all higher than with comparator.

Among the three baseline HbA1c subgroups, the success rates of the composite endpoint with VM in the HbA1c $\leq 8 \%(\mathrm{OR} 2.17,95 \% \mathrm{CI}$ $1.60-2.93),>8-9 \% \quad(\mathrm{OR} \quad 1.88, \quad 95 \% \quad \mathrm{CI}$ $1.18-2.99)$, and $>9 \% \quad(\mathrm{OR} 2.02, \quad 95 \% \quad \mathrm{CI}$ 1.17-3.48) subgroups were all higher than with comparator.

Results of the composite endpoint from the non-prespecified subgroups analysis after PMS are shown in Supplementary Fig. 2. The success rates of the composite endpoint with VM in the age (except $>75$ years old), gender, duration of T2DM, complications, smoking history, and T2DM family history (except in unclear T2DM family history) subgroups were all higher than with comparator.

\section{Analyses Based on IM and AM Subgroups of Comparator Cohort}

The success rate of the composite endpoint and the proportion of patients reaching $\mathrm{HbA1c}<$ $7 \%$ with VM and IM were compared after PSM (Fig. $4 a, b)$. The success rate of the composite endpoint was $48.1 \%(154 / 320)$ with VM and $31.6 \%(101 / 320)$ with IM at 12 months $(P<0.001 ;$ OR $2.01,95 \%$ CI $1.45-2.77)$. The proportions of patients with HbA1c $<7 \%$ were similar between the two cohorts at 3 months [40.5\% (115/284) with VM and 41.5\% (125/301) with IM]. The proportion increased steadily over time in the VM cohort, but not in the IM cohort. At 12 months, the proportion of patients reaching HbA1c $<7 \%$ with VM $(50.6 \%$, $162 / 320$ ) was significantly higher than with IM (39.7\%, 127/320) $(P<0.01)$.

The success rate of the composite endpoint and the proportion of patients reaching 
A

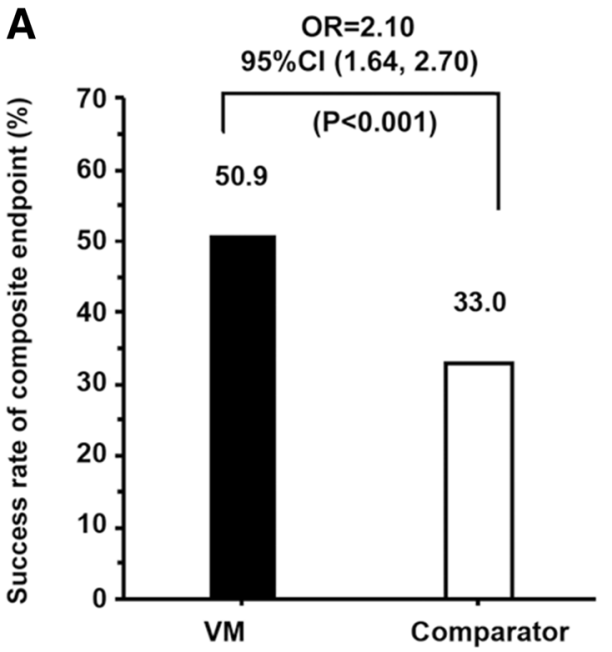

B

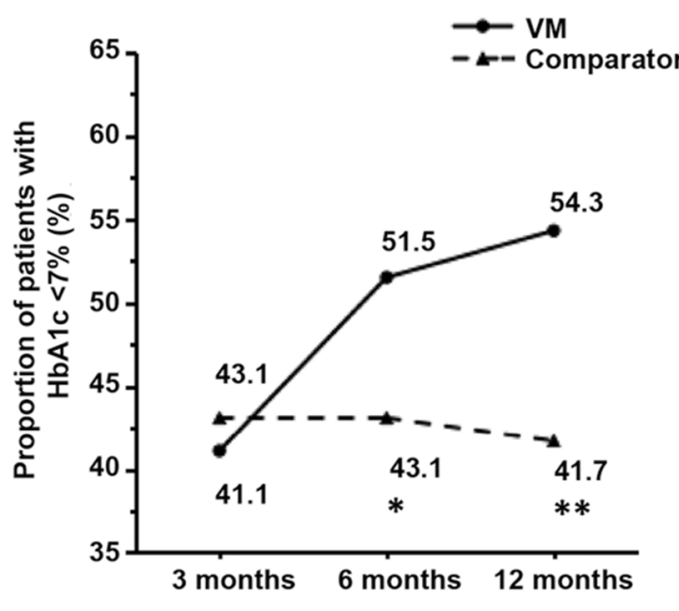

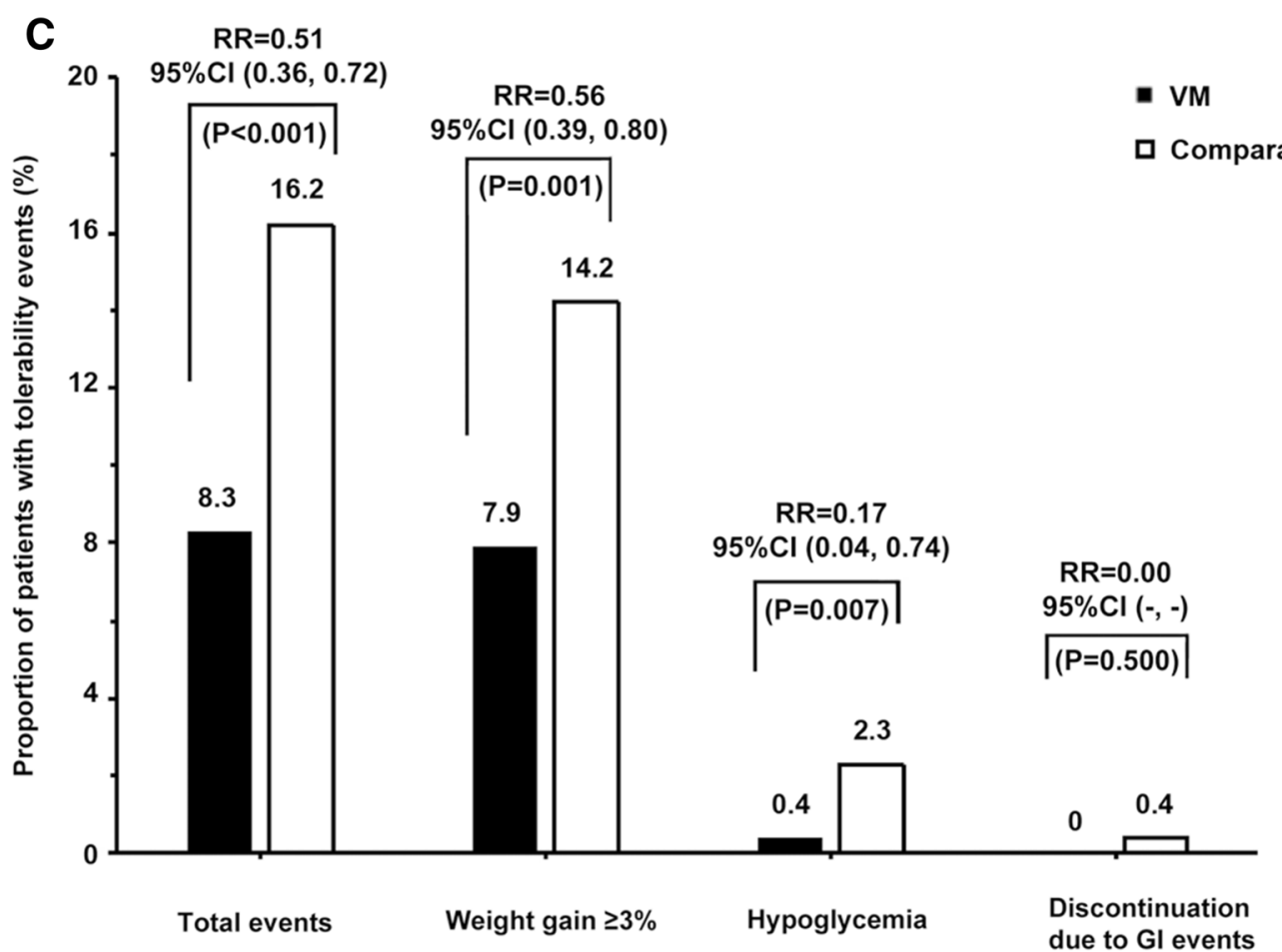

Fig. 2 Composite primary endpoint and individual components of the composite endpoint in the vildagliptin addon to metformin (VM) cohort and dual oral antidiabetes agent, non-vildagliptin combination therapy (comparator) cohort after propensity score matching $(n=530$ per cohort, full analysis set). a Success rate of composite endpoint. b Proportion of patients with HbAlc $<7 \%$.

HbA1c $<7 \%$ were compared between the VM and AM cohorts after PSM (Fig. 4c, d). The success rate of the composite endpoint was $56.7 \%$ c Proportion of patients with tolerability events. Success was defined as $\mathrm{HbAlc}<7 \%$ and without any tolerability event [hypoglycemia, weight gain $\geq 3 \%$ and discontinuation due to gastrointestinal events]. ${ }^{*} P<0.01$, ${ }^{* *} P<0.001$, VM vs. comparator. OR odds ratio, $\mathrm{RR}$ relative risk

(89/157) with VM and 45.9\% (72/157) with AM at 12 months $(P=0.055$; OR $1.54,95 \%$ CI 0.99-2.40). There were no significant 


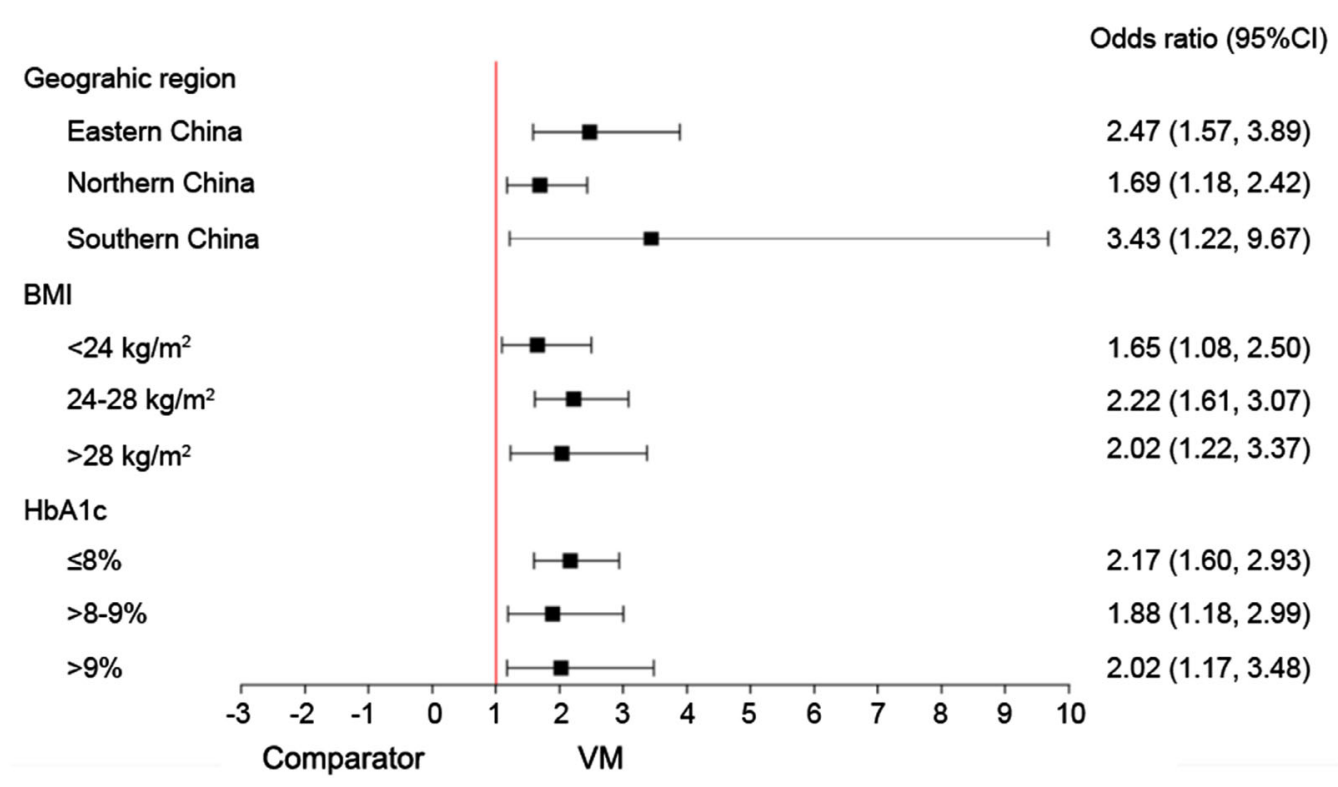

Fig. 3 Subgroup analysis of composite endpoint before propensity score matching (full analysis set). Success was defined as $\mathrm{HbAlc}<7 \%$ and without any tolerability

differences in the proportion of patients with $\mathrm{HbA} 1 \mathrm{c}<7 \%$ at 3, 6, or 12 months between the two cohorts (all $P>0.05$ ), but a trend of improved HbA1c control was observed for VM throughout the treatment period (3 months: $48.1 \%$ vs. $43.9 \%$; 6 months: $57.8 \%$ vs. $49.4 \%$, 12 months: $58.6 \%$ vs. $50.3 \%$ ).

\section{Safety}

Data on the occurrence of $\mathrm{AE}$ leading to treatment discontinuation in the safety set are shown in Table 2. The rates of any AE were 8.3\% $(60 / 724)$ and $12.8 \%(99 / 771)$ in the $\mathrm{VM}$ and comparator cohorts, respectively. Hypoglycemia occurred in $0.3 \%(2 / 724)$ and $2.3 \%$ $(18 / 771)$ in the VM and comparator cohorts, respectively.

\section{DISCUSSION}

The China PDS was a post-marketing, multicenter, prospective, observational, real-world study. The results showed that patients in the VM cohort had better glycemic control than the comparator cohort. In addition, the occurrence event (hypoglycemia, weight gain $\geq 3 \%$, and discontinuation due to gastrointestinal events)

of tolerability events (hypoglycemia and weight gain $\geq 3 \%$ ) in the $\mathrm{VM}$ cohort was significantly lower than in the comparator cohort after PSM.

As highlighted by the guidelines, T2DM treatment should be individualized according to each patient on the basis of desired efficacy, tolerance, safety, and treatment costs [8]. In the present real-world study in China, our pre-PSM baseline showed that add-on vildagliptin was preferentially prescribed in patients with higher BMI, consistent with the EDGE study, a worldwide real-world study based on 43,791 patients with T2DM [17]. Notably, the BMI in the VM cohort was lower than in other real-world studies [17-20]. This could be due to the threshold for overweight in Asians being lower than in other populations, especially in Caucasians [21, 22]. Furthermore, patients who received VM were younger, and had a shorter duration of T2DM and a higher baseline HbA1c in the present study, while no such trend was observed in the EDGE study [17]. Notably, a regional difference in baseline characteristics was observed in the EDGE study, with younger age and shorter duration of disease reported in the developing countries and a relatively low baseline HbA1c reported for the East Asian 

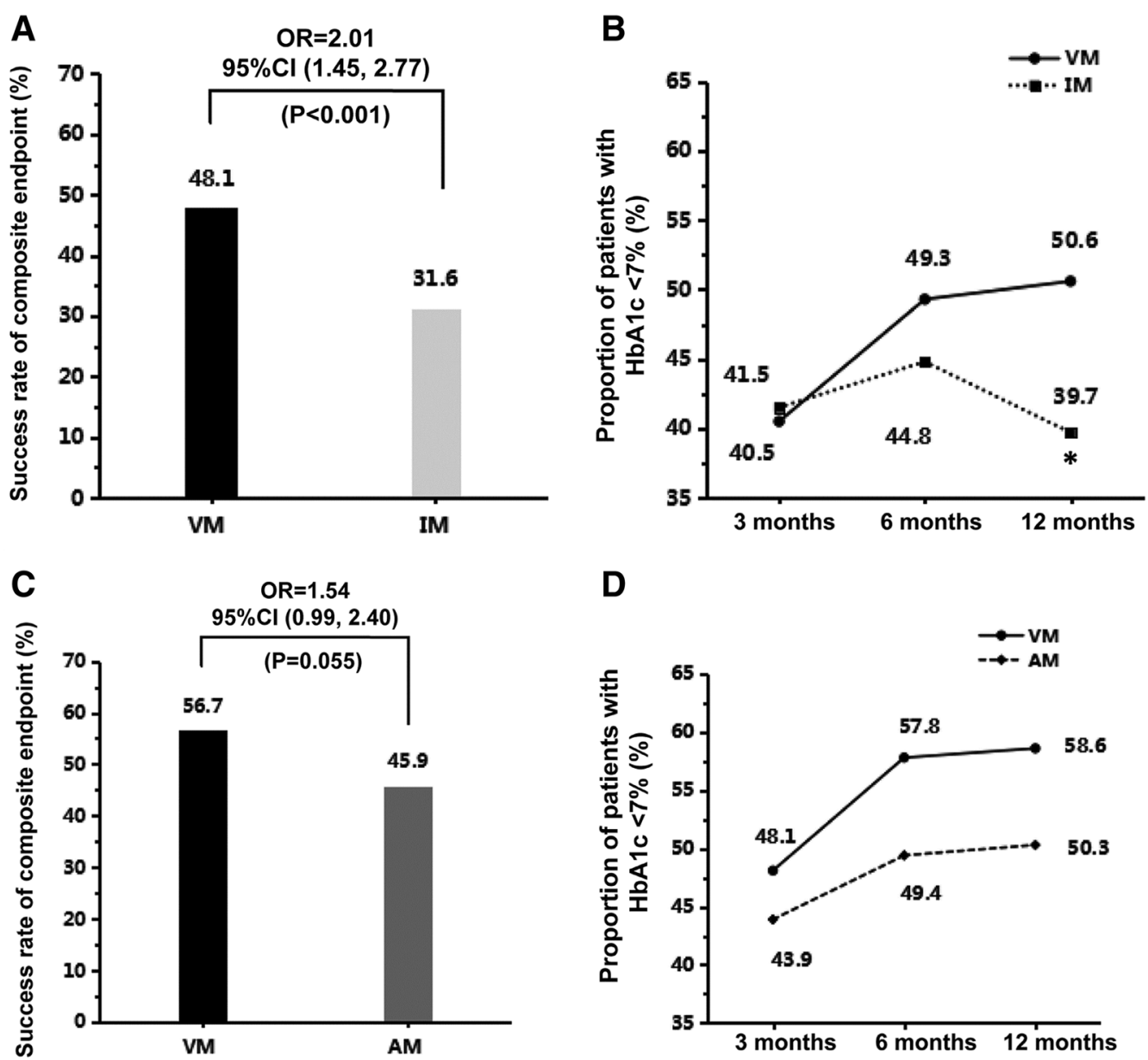

Fig. 4 Composite endpoint and proportion of patients with $\mathrm{HbAlc}<7 \%$ in the vildagliptin add-on to metformin (VM) cohort and the $\alpha$-glucosidase inhibitor addon to metformin (AM) and insulin-secreting agent add-on to metformin (IM) cohorts after propensity score matching (full analysis set). a, b Success rate of composite endpoint and proportion of patients with $\mathrm{HbA} 1 \mathrm{c}<7 \%$ in

population (South Korea and Philippines) [23]. The baseline characteristics for our Chinese T2DM population were in general agreement with the profile reported for East Asians in the EDGE study [23], suggesting an earlier onset of disease and early treatment intensification in the Chinese T2DM population. The differences in prescription patterns could be due to the different prescription preference of the physicians, patient ethnicity, and disease course in Eastern and Western countries. Other reasons for the differences in prescription pattern require further research.

the VM and IM cohorts ( $n=320$ per cohort). $\mathbf{c}$, $\mathbf{d}$ Success rate of composite endpoint and proportion of patients with HbAlc $<7 \%$ in the VM and AM cohorts $(n=157$ per cohort). Success was defined as HbAlc $<7 \%$ and without any tolerability event (hypoglycemia, weight gain $\geq 3 \%$, and discontinuation due to gastrointestinal events). ${ }^{*} P<0.01$, VM vs. IM. OR odds ratio

Several studies have shown that the addition of vildagliptin to either high- or low-dose metformin provides superior efficacy compared with monotherapy and with an overall comparable tolerability profile. The potential dosesparing effect of adding vildagliptin to low-dose metformin is preferred to an increased dose of metformin [4, 7]. This allows patients to achieve equivalent or superior HbA1c-lowering effects without the GI tolerability issues associated with higher doses of metformin [24]. The EDGE study showed that after 12 months of treatment, $35 \%$ of patients achieved the glycemic 
Table 2 Adverse events in the VM and comparator cohorts (occurrence rate $>0.5 \%$, safety analysis set)

\begin{tabular}{|c|c|c|c|c|}
\hline \multirow[t]{2}{*}{ Preferred terms in MedDRA classification system } & \multicolumn{2}{|c|}{$\mathrm{VM}(n=724)$} & \multicolumn{2}{|c|}{ Comparator $(n=771)$} \\
\hline & $n(\%)$ & Event frequency & $\overline{n(\%)}$ & Event frequency \\
\hline Any adverse event (overall) & $60(8.3)$ & 79 & $99(12.8)$ & 131 \\
\hline Metabolic and nutritional disorders & $2(0.3)$ & 2 & $23(3.0)$ & 29 \\
\hline Hypoglycemia & $2(0.3)$ & 2 & $18(2.3)$ & 23 \\
\hline Infections and infestations & $15(2.1)$ & 22 & $18(2.3)$ & 23 \\
\hline Upper respiratory tract infection & $8(1.1)$ & 10 & $8(1.0)$ & 11 \\
\hline Investigations & $11(1.5)$ & 11 & $8(1.0)$ & 9 \\
\hline Abnormal blood glucose & $9(1.2)$ & 9 & $6(0.8)$ & 7 \\
\hline Nervous system disorders ${ }^{\mathrm{a}}$ & $3(0.4)$ & 4 & $7(0.9)$ & 8 \\
\hline Injury, poisoning, and procedural complications & $3(0.4)$ & 3 & $5(0.6)$ & 5 \\
\hline Musculoskeletal and connective tissue disorders & $4(0.6)$ & 4 & $5(0.6)$ & 5 \\
\hline Skin and subcutaneous tissue disorders & $6(0.8)$ & 6 & $4(0.5)$ & 4 \\
\hline Gastrointestinal disorders & $8(1.1)$ & 8 & $11(1.4)$ & 11 \\
\hline Cardiac disorders & $1(0.1)$ & 1 & $10(1.3)$ & 12 \\
\hline Eye disorders & $6(0.8)$ & 7 & $5(0.6)$ & 6 \\
\hline
\end{tabular}

VM vildagliptin add-on to metformin

${ }^{a}$ Three cases each of stroke occurred in the VM and comparator cohorts

goal on a vildagliptin-based combination treatment, which was better than those receiving other dual OAD treatment (23\%) [17] and that a consistent favorable HbA1c response was observed across all regions [23]. A 2-year followup RCT of 3118 patients with T2DM also showed that vildagliptin was more effective in achieving a composite endpoint of HbA1c $<7 \%$ without hypoglycemia or weight gain $(29.8 \%)$ compared with glimepiride as add-on to metformin (19.4\%) [21]. Despite the different research designs, follow-up, and inclusion criteria, these studies all showed that the control of blood glucose after VM treatment was better than that after other dual OAD treatment. Our results further extended these previous observations by showing that VM was more effective than dual OAD non-vildagliptin combinations for T2DM in a real-world Chinese population. Notably, the present study showed higher success rates for the composite endpoint in both the VM and comparator cohorts than in the other studies $[17,21]$, which might be due to the relatively shorter duration of T2DM (around 4-5 years) in the present study.

In the subgroup analysis, we found that the success rate of the composite endpoint in the VM cohort was significantly higher than in the IM cohort and that the proportion of patients with glycemic control was retained for the VM cohort after 6 months, but not for the IM cohort. This suggests that the effectiveness of VM was better than for IM treatment in clinical practice, which is in line with the published RCTs $[22,25,26]$. Among patients treated with IM, $76.7 \%(250 / 326)$ were on SU add-on to metformin and the diminished glycemic response observed in the IM cohort might be partially attributed to the suboptimal dose of SUs (due to fear of hypoglycemia) and the relatively longer duration of T2DM for the IM cohort [27]. Indeed, the duration of glycemic 
lowering effect of SUs decreased with the duration of T2DM as a result of progressive deterioration in $\beta$-cell function. On the other hand, vildagliptin targets $\alpha$-cells in addition to $\beta$-cells and may provide a prolonged glycemic response [28]. The shorter durability of SUs as add-on to monotherapy compared with vildagliptin has also been reported in the EDGE study [29]. Nevertheless, one large-scale retrospective cohort study reported improved durability of glycemic response with SUs versus DPP-4 inhibitors as add-on to metformin; the differences might be attributed to the differences in baseline BMI, HbA1c, and T2DM duration [30].

In addition, guidelines for T2DM management also recommend avoiding side effects, particularly hypoglycemia and weight gain, when physicians make decisions about which antidiabetes drug to choose $[4,7,8]$. In China, the most common dual therapy is insulin-secreting agent combined with metformin [31]. This might be the main reason for the high incidence of hypoglycemia and weight gain in patients with T2DM in China. Hypoglycemia hampers effective glycemic control and may lead to the occurrence of serious complications including cardiovascular events [8]. Severe hypoglycemia is also a key cause of death and disability in patients with T2DM. Hence, hypoglycemia is an important index for the clinical evaluation of OADs [32]. The EDGE study showed that the incidence of hypoglycemia in the VM cohort $(0.23 \%)$ was lower than in the dual OAD comparator cohort (1.28\%) and the results of the present study are in line with the EDGE study [33]. Indeed, in the present study, the incidence of hypoglycemia in the VM cohort $(0.4 \%)$ was significantly lower than in the comparator cohort $(2.3 \%)$. Of course, hypoglycemia events may be distressful and even represent some danger to the patient. Treatments associated with a lower risk of hypoglycemia events should be preferred in order to improve patient experience and safety [4, 7]. In a previous RCT, vildagliptin as add-on to metformin versus other drug combined with metformin led to markedly lower risks of hypoglycemia [34]. Taken together, the literature and the present study suggest that vildagliptin is safe regarding the occurrence of hypoglycemia in clinical practice.
Weight loss is well established in the prevention of T2DM and is a critical component of T2DM management. Unfortunately, many traditional glucose-lowering drugs can lead to weight gain, which has a negative impact on the management of T2DM for most patients $[35,36]$. Previous studies have shown that VM, compared to monotherapy, was more effective in reducing HbA1c levels and without weight gain $[24,37]$. We found that the occurrence of $\geq 3 \%$ weight gain in the VM cohort was significantly lower than in the comparator cohort. This suggested the clinical benefits of VM treatment on body weight control compared to other non-vildagliptin dual OAD therapies.

GI events are common complications that occur during T2DM treatment. The most common untoward effects of $\alpha$-glucosidase inhibitors in T2DM are GI events such as bowel gastroparesis, abdominal distention, abdominal pain, diarrhea, and increased GI exhaustion [38-40]. Moreover, in China, $10.9 \%$ of patients with T2DM receive $\alpha$-glucosidase inhibitor and metformin therapy [31], which may increase or aggravate GI events. As a DPP-4 inhibitor, vildagliptin shows a good glycemic control effect while it did not increase GI events in patients with T2DM in previous RCT studies [40]. In the present study, we did not observe any discontinuation due to GI events, while the occurrence of GI disorders in the SAS population was similar. These low rates of occurrence of GI events are consistent with the results from the EDGE study (1.45\% vs. $1.28 \%$ ) [17], supporting that combination therapy is tolerable in a realworld clinical practice.

We also found that the rate of overall AEs in the VM cohort $(8.3 \%)$ was lower than in the comparator cohort (12.8\%). Similarly, a 24-week, randomized, multicenter, crossover study in Germany showed that fewer patients with AEs were reported with VM (15\%) than with other dual OAD treatment (37.1\%) [41]. In the present study, the frequencies of AEs were lower than that in the German study [41], which might be explained by differences in study design. Indeed, in RCTs, there is usually a higher compliance than in real-world studies resulting from the stricter and regular follow-up and supervision. Whereas in real-world studies, 
the AEs rates are generally lower because there is no formal enquiry and they are reported by the patients themselves. Hence, the EDGE study reported that the rate of AEs was only $5.28 \%$ in the vildagliptin add-on to metformin treatment cohort [17]. In the present study, no difference in cardiac disorders (VM vs. comparator, $0.1 \%$ vs. $1.3 \%$ ) or nervous system disorders (including stroke; VM vs. comparator, $0.4 \%$ vs. $0.9 \%$ ) was observed between the $\mathrm{VM}$ and comparator cohorts, which was consistent with a metaanalysis of 17,446 patients with T2DM that showed that vildagliptin was not associated with an increased risk of major adverse cardiovascular events or heart failure compared with the comparators [42]. In addition, a recent trial in patients with T2DM and heart failure also showed that vildagliptin was not associated with decreased left ventricular ejection fraction, worsening of heart failure, or hospitalization due to heart failure, compared with placebo [43].

This is a real-world study and therefore has some limitations. Because of no randomization in our study, there were biases and confounding factors that should be addressed. The main limitation of the study was the selection bias from the physician being responsible for selecting treatment. The treatment decisions were based on several factors and demographic characteristics, which included age, gender, BMI, ethnicity, baseline HbA1c levels, duration of T2DM, complications, smoking history, and T2DM family history. These factors may have influenced not only the choice of treatment but also the results of the study, especially in terms of safety assessment. Matching or stratified sampling might help to control these relevant factors. On the other hand, as a result of the difference of prescription patterns in the realworld setting, an important difference in age and disease duration was observed in the VM versus comparator cohorts. To preserve an adequate sample size for analysis, these factors was not adjusted in the PSM. Only a small number of patients received vildagliptin with other OADs and they were not analyzed in the present study because PSM would not have been possible. Combinations of vildagliptin with OADs like pioglitazone and glimepiride have been reported to be efficient and safe in patients with T2DM in clinical trials [44, 45]. Future studies will have to examine the combination of vildagliptin with OADs other than metformin in a real-world setting.

\section{CONCLUSION}

In summary, the present real-world study from China shows that compared with dual OAD non-vildagliptin combination therapies, vildagliptin add-on to metformin is effective, well tolerated, and safe for the treatment of Chinese patients with T2DM.

\section{ACKNOWLEDGEMENTS}

The authors acknowledge all the patients and physicians participating in this study. We acknowledge the ethics committee of Chinese PLA General Hospital for piloting the ethical review of this study.

Funding. This study and the article processing charges were funded by Novartis. All authors had full access to all of the data in this study and take complete responsibility for the integrity of the data and accuracy of the data analysis.

Authorship. All named authors meet the International Committee of Medical Journal Editors (ICMJE) criteria for authorship for this article, take responsibility for the integrity of the work as a whole, and have given their approval for this version to be published.

Disclosures. Li Zang, Yin Han, Lixian Chen, Daqing Hu, Hui Jin, Nailong Yang, Xiaoyun Shi, Linlang Liang, Quanmin Li, and Yiming $\mathrm{Mu}$ declare that they have nothing to disclose. Mingming Liu is an employee of Novartis China. Hong Fan is an employee of Novartis China.

Compliance with Ethics Guidelines. This study was conducted between June 2013 and April 2017 in 52 centers of China. The study was 
independently approved by each ethics committee/institutional review board of each study center (Supplementary Table 1). Chinese PLA General Hospital piloted the ethical review of this study. Written informed consent for the study was obtained from all participants. Since data on individual people in any form were not included in this manuscript, the consent for publication was waived by the ethics committees. The study was conducted in compliance with the Helsinki Declaration of 1964 and its later amendments.

Data Availability. All data generated or analyzed during this study are included in this published article/as supplementary information files.

Open Access. This article is distributed under the terms of the Creative Commons Attribution-NonCommercial 4.0 International License (http://creativecommons.org/licenses/ by-nc/4.0/), which permits any noncommercial use, distribution, and reproduction in any medium, provided you give appropriate credit to the original author(s) and the source, provide a link to the Creative Commons license, and indicate if changes were made.

\section{REFERENCES}

1. Wang L, Gao P, Zhang M, et al. Prevalence and ethnic pattern of diabetes and prediabetes in China in 2013. JAMA. 2017;317(24):2515-23. https://doi. org/10.1001/jama.2017.7596.

2. Ji L, Hu D, Pan C, et al. Primacy of the $3 B$ approach to control risk factors for cardiovascular disease in type 2 diabetes patients. Am J Med. 2013;126(10):925 e911-22. https://doi.org/10. 1016/j.amjmed.2013.02.035.

3. Zhang L, Ji L, Guo L, et al. Treatment patterns and glycemic control in older adults with type 2 diabetes mellitus receiving only oral antidiabetes drugs in China. Diabetes Technol Ther. 2015;17(11):816-24. https://doi.org/10.1089/dia. 2015.0094 .

4. American Diabetes Association. Standards of medical care in diabetes-2014. Diabetes Care. 2014;37(Supplement 1):S14-80.
5. Turner RC, Cull CA, Frighi V, Holman RR. Glycemic control with diet, sulfonylurea, metformin, or insulin in patients with type 2 diabetes mellitus: progressive requirement for multiple therapies (UKPDS 49). UK Prospective Diabetes Study (UKPDS) Group. JAMA. 1999;281(21):2005-12.

6. Ito $\mathrm{H}$, Ishida $\mathrm{H}$, Takeuchi $\mathrm{Y}$, et al. Long-term effect of metformin on blood glucose control in nonobese patients with type 2 diabetes mellitus. Nutr Metab. 2010;7:83. https://doi.org/10.1186/17437075-7-83.

7. Chinese Diabetes Society. China guideline for type 2 diabetes 2017. Chin J Diabetes Mellitus. 2018;10(1):4-67. https://doi.org/10.3760/cma.j. issn.1674-5809.2018.01.003

8. Garber AJ, Abrahamson MJ, Barzilay JI, et al. Consensus Statement by the American Association of Clinical Endocrinologists and American College of Endocrinology on the comprehensive type 2 Diabetes Management Algorithm-2019 executive summary. Endocr Pract. 2019;25(1):69-100. https:// doi.org/10.4158/CS-2018-0535.

9. Foley JE, Jordan J. Weight neutrality with the DPP-4 inhibitor, vildagliptin: mechanistic basis and clinical experience. Vasc Health Risk Manag. 2010;6:541-8.

10. Ahren B, Foley JE, Bosi E. Clinical evidence and mechanistic basis for vildagliptin's action when added to metformin. Diabetes Obes Metab. 2011;13(3):193-203. https://doi.org/10.1111/j. 1463-1326.2010.01321.x.

11. Ahren B, Foley JE, Ferrannini E, et al. Changes in prandial glucagon levels after a 2-year treatment with vildagliptin or glimepiride in patients with type 2 diabetes inadequately controlled with metformin monotherapy. Diabetes Care. 2010;33(4):730-2. https://doi.org/10.2337/dc091867.

12. Filozof C, Gautier JF. A comparison of efficacy and safety of vildagliptin and gliclazide in combination with metformin in patients with type 2 diabetes inadequately controlled with metformin alone: a 52-week, randomized study. Diabet Med. 2010;27(3):318-26. https://doi.org/10.1111/j.14645491.2010.02938.x.

13. Pan C, Xing X, Han P, et al. Efficacy and tolerability of vildagliptin as add-on therapy to metformin in Chinese patients with type 2 diabetes mellitus. Diabetes Obes Metab. 2012;14(8):737-44. https:// doi.org/10.1111/j.1463-1326.2012.01593.x.

14. Wing RR, Lang W, Wadden TA, et al. Benefits of modest weight loss in improving cardiovascular risk factors in overweight and obese individuals with 
type 2 diabetes. Diabetes Care. 2011;34(7):1481-6. https://doi.org/10.2337/dc10-2415.

15. Alborzi P, Patel N, Agarwal R. Home blood pressures are of greater prognostic value than hemodialysis unit recordings. Clin J Am Soc Nephrol. 2007;2(6):1228-34. https://doi.org/10.2215/CJN. 02250507 .

16. Holmskov M, Storebo OJ, Moreira-Maia CR, et al. Gastrointestinal adverse events during methylphenidate treatment of children and adolescents with attention deficit hyperactivity disorder: a systematic review with meta-analysis and Trial Sequential Analysis of randomised clinical trials. PLoS One. 2017;12(6):e0178187. https://doi.org/10.1371/ journal.pone.0178187.

17. Mathieu C, Barnett AH, Brath $\mathrm{H}$, et al. Effectiveness and tolerability of second-line therapy with vildagliptin vs other oral agents in type 2 diabetes: a reallife worldwide observational study (EDGE). Int J Clin Pract. 2013;67(10):947-56. https://doi.org/10. 1111/ijcp.12252.

18. Ayvaz G, Keskin L, Akin F, et al. Real-life safety and efficacy of vildagliptin as add-on to metformin in patients with type 2 diabetes in Turkey-GALATA study. Curr Med Res Opin. 2015;31(4):623-32. https://doi.org/10.1185/03007995.2015.1019609.

19. Melzer Cohen C, Davis C, Shalev V, Chodick G. Effectiveness of vildagliptin as add-on to metformin monotherapy among uncontrolled type 2 diabetes mellitus patients in a real-world setting. J Diabetes. 2018;10(1):68-72. https://doi.org/10.1111/17530407.12560 .

20. Shelbaya S, Rakha S. Effectiveness and safety of vildagliptin and vildagliptin add-on to metformin in real-world settings in Egypt-results from the GUARD study. Curr Med Res Opin. 2017;33(5):797-801. https://doi.org/10.1080/ 03007995.2016 .1277199$.

21. Bader G, Geransar P, Schweizer A. Vildagliptin more effectively achieves a composite endpoint of $\operatorname{HbA}(1) \mathrm{c}<70 \%$ without hypoglycaemia and weight gain compared with glimepiride after 2 years of treatment. Diabetes Res Clin Pract. 2013;100(3):e78-81. https://doi.org/10.1016/j. diabres.2013.03.011.

22. Hirose T, Suzuki M, Tsumiyama I. Efficacy and safety of vildagliptin as an add-on to insulin with or without metformin in Japanese patients with type 2 diabetes mellitus: a 12-week, double-blind, randomized study. Diabetes Ther. 2015;6(4):559-71. https://doi.org/10.1007/s13300-015-0147-6.

23. Brath H, Paldanius PM, Bader G, Kolaczynski WM, Nilsson PM. Differences in glycemic control across world regions: a post hoc analysis in patients with type 2 diabetes mellitus on dual antidiabetes drug therapy. Nutr Diabetes. 2016;6(7):e217. https://doi. org/10.1038/nutd.2016.25.

24. Bosi E, Dotta F, Jia Y, Goodman M. Vildagliptin plus metformin combination therapy provides superior glycaemic control to individual monotherapy in treatment-naive patients with type 2 diabetes mellitus. Diabetes Obes Metab. 2009;11(5):506-15. https://doi.org/10.1111/j.1463-1326.2009.01040.x.

25. Otowa-Suematsu N, Sakaguchi K, Nakamura T, et al. Comprehensive evaluation of combination therapy with basal insulin and either lixisenatide or vildagliptin in Japanese patients with type 2 diabetes: a randomized, open-label, parallel-group, multicenter study. Diabetes Ther. 2018;9(5):2067-79. https://doi.org/10.1007/s13300-018-0505-2.

26. Forst T, Koch C, Dworak M. Vildagliptin versus insulin in patients with type 2 diabetes mellitus inadequately controlled with sulfonylurea: results from a randomized, 24 week study. Curr Med Res Opin. 2015;31(6):1079-84. https://doi.org/10.1185/ 03007995.2015 .1039936$.

27. Ahren B, Mathieu C, Bader G, Schweizer A, Foley JE. Efficacy of vildagliptin versus sulfonylureas as addon therapy to metformin: comparison of results from randomised controlled and observational studies. Diabetologia. 2014;57(7):1304-7. https:// doi.org/10.1007/s00125-014-3222-z.

28. Ahren B, Foley JE. Improved glucose regulation in type 2 diabetic patients with DPP-4 inhibitors: focus on alpha and beta cell function and lipid metabolism. Diabetologia. 2016;59(5):907-17. https://doi. org/10.1007/s00125-016-3899-2.

29. Brath H, Paldanius PM, Bader G, Mathieu C. Relationship between duration of type 2 diabetes and effectiveness of DPP-4 inhibitor versus sulfonylurea as add-on therapy: a post hoc analysis. Diabetes Ther. 2017;8(4):829-36. https://doi.org/10.1007/ s13300-017-0276-1.

30. Mamza J, Mehta R, Donnelly R, Idris I. Important differences in the durability of glycaemic response among second-line treatment options when added to metformin in type 2 diabetes: a retrospective cohort study. Ann Med. 2016;48(4):224-34. https:// doi.org/10.3109/07853890.2016.1157263.

31. Ji L, Lu J, Weng J, et al. China type 2 diabetes treatment status survey of treatment pattern of oral drugs users. J Diabetes. 2015;7(2):166-73. https:// doi.org/10.1111/1753-0407.12165.

32. Inzucchi SE, Bergenstal RM, Buse JB, et al. Management of hyperglycaemia in type 2 diabetes: a patient-centered approach. Position statement of 
the American Diabetes Association (ADA) and the European Association for the Study of Diabetes (EASD). Diabetologia. 2012;55(6):1577-96. https:// doi.org/10.1007/s00125-012-2534-0.

33. Mendivil CO, Marquez-Rodriguez E, Angel ID, et al. Comparative effectiveness of vildagliptin in combination with other oral anti-diabetes agents in usual-care conditions: the EDGE-Latin America study. Curr Med Res Opin. 2014;30(9):1769-76. https://doi.org/10.1185/03007995.2014.928274.

34. Matthews DR, Dejager S, Ahren B, et al. Vildagliptin add-on to metformin produces similar efficacy and reduced hypoglycaemic risk compared with glimepiride, with no weight gain: results from a 2-year study. Diabetes Obes Metab. 2010;12(9):780-9. https://doi.org/10.1111/j.1463-1326.2010.01233.x.

35. Van Gaal L, Scheen A. Weight management in type 2 diabetes: current and emerging approaches to treatment. Diabetes Care. 2015;38(6):1161-72. https://doi.org/10.2337/dc14-1630.

36. Nunes AP, Iglay K, Radican L, et al. Hypoglycaemia seriousness and weight gain as determinants of cardiovascular disease outcomes among sulfonylurea users. Diabetes Obes Metab. 2017;19(10):1425-35. https://doi.org/10.1111/ dom.13000.

37. Filozof C, Schwartz S, Foley JE. Effect of vildagliptin as add-on therapy to a low-dose metformin. World J Diabetes. 2010;1(1):19-26. https://doi.org/10.4239/ wjd.v1.i1.19.

38. Sun F, Chai S, Yu K, et al. Gastrointestinal adverse events of glucagon-like peptide- 1 receptor agonists in patients with type 2 diabetes: a systematic review and network meta-analysis. Diabetes Technol Ther. 2015;17(1):35-42. https://doi.org/10.1089/dia. 2014.0188.

39. Horowitz M, Aroda VR, Han J, Hardy E, Rayner CK. Upper and/or lower gastrointestinal adverse events with glucagon-like peptide-1 receptor agonists: incidence and consequences. Diabetes Obes Metab. 2017;19(5):672-81. https://doi.org/10.1111/dom. 12872 .

40. Wu S, Chai S, Yang J, et al. Gastrointestinal adverse events of dipeptidyl peptidase 4 inhibitors in type 2 diabetes: a systematic review and network metaanalysis. Clin Ther. 2017;39(9):1780-9.e33. https:// doi.org/10.1016/j.clinthera.2017.07.036.

41. Ludemann J, Dutting ED, Dworak M. Patient preference and tolerability of a DPP-4 inhibitor versus a GLP-1 analog in patients with type 2 diabetes mellitus inadequately controlled with metformin: a 24-week, randomized, multicenter, crossover study. Ther Adv Endocrinol Metab. 2015;6(4):141-8. https://doi.org/10.1177/2042018815595584.

42. McInnes G, Evans M, Del Prato S, et al. Cardiovascular and heart failure safety profile of vildagliptin: a meta-analysis of 17000 patients. Diabetes Obes Metab. 2015;17(11):1085-92. https://doi.org/10. 1111/dom.12548.

43. McMurray JJV, Ponikowski P, Bolli GB, et al. Effects of vildagliptin on ventricular function in patients with type 2 diabetes mellitus and heart failure: a randomized placebo-controlled trial. JACC Heart Fail. 2018;6(1):8-17. https://doi.org/10.1016/j.jchf. 2017.08.004.

44. Garber AJ, Schweizer A, Baron MA, Rochotte E, Dejager S. Vildagliptin in combination with pioglitazone improves glycaemic control in patients with type 2 diabetes failing thiazolidinedione monotherapy: a randomized, placebo-controlled study. Diabetes Obes Metab. 2007;9(2):166-74. https://doi.org/10.1111/j.14631326.2006.00684.x.

45. Garber AJ, Foley JE, Banerji MA, et al. Effects of vildagliptin on glucose control in patients with type 2 diabetes inadequately controlled with a sulphonylurea. Diabetes Obes Metab. 2008;10(11):1047-56. https://doi.org/10.1111/j. 1463-1326.2008.00859.x. 